\title{
Theory of twisted trunks ${ }^{\star}$
}

\author{
P. Carlqvist ${ }^{1}$, G. F. Gahm² , and H. Kristen ${ }^{3}$
}

\author{
1 Alfvén Laboratory, Royal Institute of Technology, 10044 Stockholm, Sweden \\ 2 SCFAB, Stockholm Observatory, 10691 Stockholm, Sweden \\ 3 Precise Biometrics, Dag Hammarskjölds väg 2, 22464 Lund, Sweden
}

Received 12 November 2002 / Accepted 11 March 2003

\begin{abstract}
Using the $2.6 \mathrm{~m}$ Nordic Optical Telescope we have observed a large number of elephant trunks in several $\mathrm{H}$ II regions. Here, we present a small selection of this material consisting of a few large, well-developed trunks, and some smaller ones. We find that: (i) the well-developed trunks are made up of dark filaments and knots which show evidence of twisted structures, (ii) the trunks are connected with essentially two filamentary legs running in $\mathrm{V}$-shape, and (iii) all trunks have the maximum extinction in their heads. We advance a theory of twisted elephant trunks which is based on the presence of magnetic flux ropes in molecular clouds where hot OB stars are formed. If the rope contains a local condensation it may adopt a V-shape as the $\mathrm{H}$ II region around the hot stars expands. If, in addition, the magnetic field in the rope is sufficiently twisted, the rope may form a double helix at the apex of the V. The double helix is identified with the twisted elephant trunks. In order to illustrate the mechanisms behind the double helix we have constructed a mechanical analogy model of the magnetic flux rope in which the rope has been replaced by a bundle of elastic strings loaded by a weight. Experiments with the model clearly show that part of the bundle will transform into a double helix when the twist of the bundle is sufficiently large. We have also worked out a simple theoretical model of a mass-loaded magnetic flux rope. Numerical calculations show that a double helix will indeed form when the twist of the rope exceeds a certain critical limit. Numerical model calculations are applied to both the analogy model experiments and one of the well-developed elephant trunks. On the basis of our model we also suggest a new interpretation of the so called EGGs.

The double helix mechanism is quite general, and should be active also in other suitable environments. One such environment may be the shell of supernova remnants. Another example is the expanding bubble outlined by the North Celestial Pole Loop.
\end{abstract}

Key words. ISM: H II regions - ISM: kinematics and dynamics - ISM: magnetic fields - ISM: structure ISM: supernova remnants

\section{Introduction}

Elephant trunks are elongated, dark clouds which stand out in silhouette against a background of bright nebulosity on optical images. Usually the trunks are found in close connection with molecular clouds and $\mathrm{H}$ II regions excited by one or more young, hot stars. In general the trunks are pointing like fingers towards the hot stars, and sometimes they are decorated by bright rims. Several authors have given examples of such structures (see e.g. Minkowski 1949; Pottasch 1956; Gyul'budagyan \& Akopyan 1990; Gyul'budagyan et al. 1994; Ogura \& Sugitani 1998), and their nature has been further discussed by among others Osterbrock (1957), Menon (1962), Herbig (1974), Schneps et al. (1980) and Hester et al. (1996a).

Using the elephant trunks in the Eagle Nebula (M 16) as prominent examples Spitzer (1954) and Frieman (1954) advanced a theory of the trunks based on the Rayleigh-Taylor instability. The theory assumes that the radiation from one or several hot $\mathrm{O}$ stars heats and ionizes an initially cold gas in a

\footnotetext{
Send offprint requests to: G. F. Gahm, e-mail: gahm@astro.su.se

$\star$ Based on observations collected at the Nordic Optical Telescope, La Palma, Spain.
}

region around the stars. The resulting high pressure of the ionized gas drives an expanding shell into the surrounding cold gas so that the internal pressure is approximately balanced by the ram pressure at the outside of the shell. Later, the discoveries of stellar winds and pronounced velocity gradients along the trunks motivated modifications of this theory (Schneps et al. 1980; Pound 1998).

Another theory of the formation of elephant trunks has been suggested by Pikel'ner (1973) and Pikel'ner \& Sorochenko (1974). In this theory stellar winds from young, hot stars give rise to an enhanced pressure which drives a shell outwards through a surrounding cold gas cloud. If the initial gas cloud contains denser clumps they will move at a slower speed than the shell due to their enhanced inertia. As a result, the lagging clumps may be expected to develop into long, extended structures pointing radially inwards like the trunks do. Other variations on this theme have been developed by introducing ionization-shock fronts (Giuliani 1979) and combinations of photo-ionization and winds (Garcia-Segura \& Franco 1996).

A few years ago we found that one of the more prominent elephant trunks in the Rosette Nebula has a very intriguing internal structure (Carlqvist et al. 1998). The trunk is essentially 
built up by a number of filaments and knots. An interesting finding was that the filaments show a twisted or helical structure. Further studies have revealed that also many other trunks show clear signs of being twisted. The twisted geometry of the trunks is not easy to account for by current theory. In an effort to understand the physics behind the twisted trunks, an outline of a theory of such trunks has recently been advanced by Carlquist et al. (2002, hereafter called CGK02). The theory is based on the presence of magnetized filaments in molecular clouds where hot stars are formed. A brief summary of the theory is given in next section.

\section{Sketch of the theory}

We consider a molecular cloud in which one or more hot $\mathrm{OB}$ stars have recently formed. The stars give rise to intense radiation, powerful winds, and shocks which assist to create an $\mathrm{H}$ II region around the stars. As a result of the high pressure of the plasma formed, the $\mathrm{HII}$ region expands, and the inner part of the surrounding molecular cloud is pushed radially outwards as a dense shell. The internal pressure approximately balances the external ram pressure on the shell.

As the shell expands it is supposed to encounter magnetized filaments in the cloud. The filamentary matter is confined by magnetic fields that are more or less twisted. We shall denote such a filament a magnetic flux tube. In reality the flux tube may be composed of two or more magnetic flux strands making the tube resemble a magnetic flux rope. In our model, however, we shall for the sake of simplicity assume that the flux tube consists of only one single filament. It is of great significance to learn how the expanding shell interacts with such a flux tube. The result of the interaction must of course depend on the physical conditions in the tube. If the molecular gas in the flux tube has a constant density, which is about the same as the density of the surrounding molecular cloud, the stellar wind-induced pressure will accelerate all parts of the flux tube to the same degree. The flux tube will then be incorporated in the shell, and must hence start to move radially outwards from the hot stars more or less as a unity. Even if the flux tube was not originally perpendicular to the radial direction it will be so after being swept up by the shell. Since the magnetic field is expected to be frozen in to the flux tube matter, the magnetic flux must be maintained in the tube.

A magnetic flux tube having a density that does not vary along the tube represents of course a very special case. A more likely situation is that the density varies along the tube. We therefore consider a simple example of an inhomogeneous density distribution with a localized mass condensation of relatively high density in the middle part of the tube, and a smaller density on the flanks. To begin with we assume that the magnetic field lines in the flux tube are straight, and axially directed, implying no axial electric current. When the stellar wind-induced pressure acts upon such a magnetic flux tube the condensation will, due to its enhanced inertia, be accelerated to a less degree than the flanks. We may therefore expect the magnetic flux tube to be drawn out to a shape resembling a V, where the upper parts of the $\mathrm{V}$ connect to the expanding shell.
The condensation will be situated at the apex of the $\mathrm{V}$ pointing towards the hot stars.

If instead there exists an axial current in the flux tube considered above, the result of the impact of the stellar windinduced pressure on the tube may be drastically different. For sufficiently small currents the V-shape mentioned above is indeed maintained, but for currents above a certain critical limit a considerable change of the geometry of the flux tube will take place. For large enough currents we expect part of the flux tube, in the vicinity of the condensation, to be deformed into a double helix pointing towards the central, hot stars. The double helix is identified with the twisted elephant trunks. At the far end of the double helix, as seen from the central stars, the connecting magnetic flux tubes again form a V. Taken as a whole the magnetic flux tube now resembles a Y.

Above we have described the magnetic flux tube as a more or less homogeneous filament. This is probably a strong simplification. In reality the magnetic flux tube may to a large extent consist of a number of subfilaments of enhanced density, winding around each other in a helical geometry.

In the present paper we shall develop the theory described above in some more detail. We shall consider a simple analogy model of twisted elephant trunks, and give an account of some experiments performed with this model (Sect. 4). We shall also develop a theoretical model of the mechanism leading to the double helix, and compare the numerical results obtained from the model with both the experimental results (Sect. 5) and observational results of one of the most prominent trunks in the Rosette Nebula (Sect. 6). First, however, we shall study the structure of a few elephant trunks (Sect. 3).

\section{Observations of elephant trunks}

We have collected a large number of images of elephant trunks in HII regions using the ALFOSC camera with a narrow-band $\mathrm{H} \alpha$ filter attached to the $2.6 \mathrm{~m}$ Nordic Optical Telescope (NOT). The field of view is 6.5 , and the effective scale of the CCD-detector is 0 ". 188 per pixel. The exposure times were $30 \mathrm{~min}$. From stars in the fields we measured angular resolutions in the range $\sim 0 \prime \prime 7-1{ }^{\prime \prime} .0$. To illustrate the variety of morphologies of elephant trunks we present here a small selection of images of regions in the Rosette Nebula, IC 1805, and N 81 in the Small Magellanic Cloud (SMC). More NOT images of elephant trunks, also in other H II regions, will be presented elsewhere.

The Rosette Nebula. This region (NGC 2237-2246) constitutes one of the best examples of a young $\mathrm{H}$ II region exhibiting a rich collection of dark elephant trunks, filaments, and globules. The nebula is situated in the north-western end of an elongated, giant molecular cloud (Blitz \& Thaddeus 1980; Williams et al. 1995). At the centre of the nebula is the open star cluster NGC 2244 containing 17 hot OB stars which effectively excite the nebula. Powerful stellar winds create a high pressure plasma which blows a bubble in the surrounding molecular cloud. The distance to NGC 2244, and hence to the nebula, has been estimated by several authors to be in the interval 1.4-1.6 kpc. Hensberge et al. (2000) have recently arrived at the value $1.39 \pm 0.1 \mathrm{kpc}$ which we shall adopt here. 


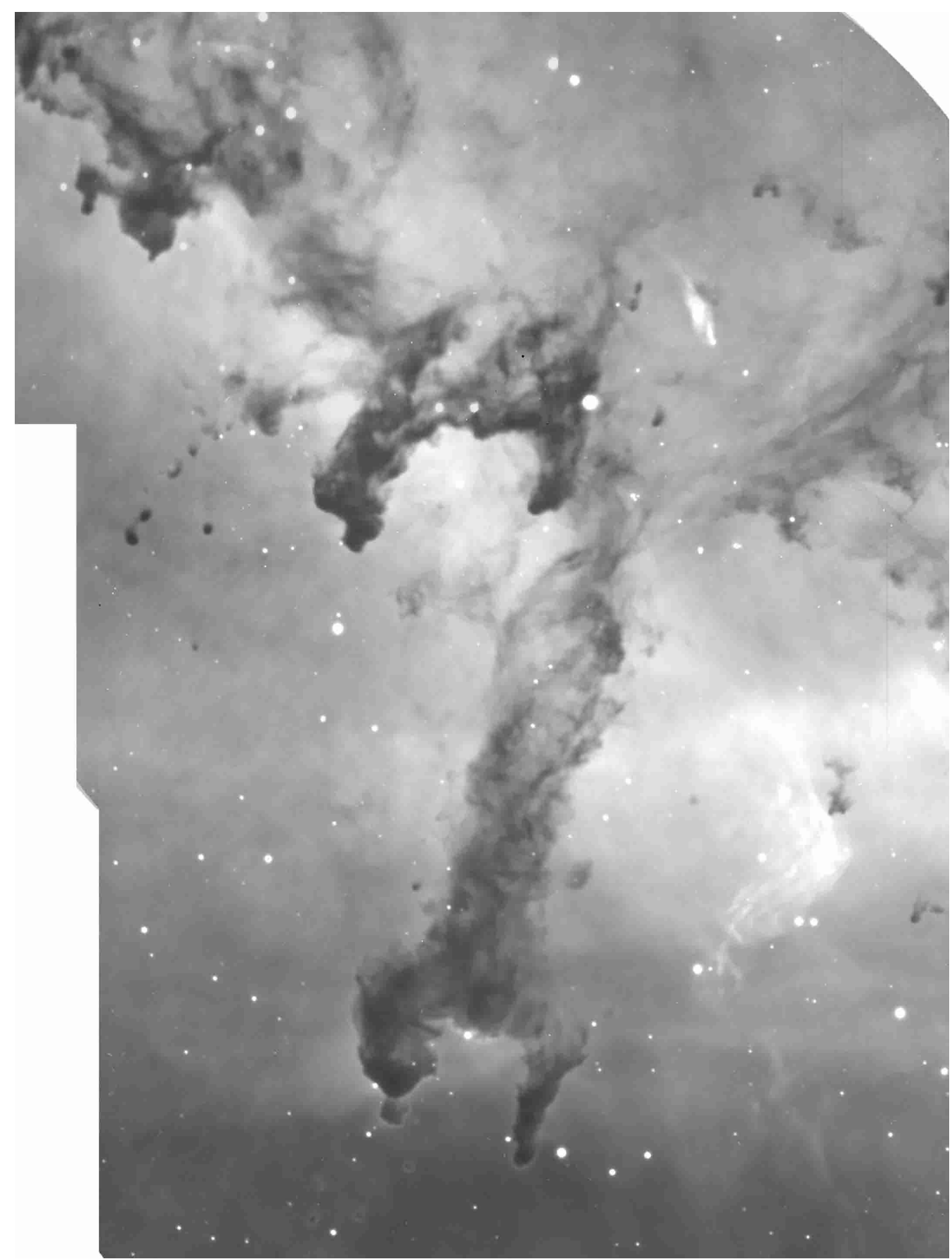

Fig. 1. Elephant trunks and dark filaments in the northern part of the Rosette Nebula. The Wrench Trunk extends southwards from just above the centre of the frame. The main body of the trunk is made up of a number of dark filaments which appear to be twisted. To the left is a string of small $\left(<6^{\prime \prime}\right)$ globules, probably constituting the visible remains of a former trunk. The image covers $405^{\prime \prime} \times 540^{\prime \prime}$. North is up.

In Fig. 1 we show a small part of the Rosette nebula. The frame is centered about $12^{\prime}$ north of the centre of NGC 2244 , and is a mosaic image based on four individual images obtained with the NOT. Most of the dark structures are fairly well ordered, and form elongated, and partly coherent filaments. Of particular interest here are the elephant trunks, which point radially inwards towards NGC 2244 (situated below the frame). A number of dark filaments also exist which run in other directions. Many of these filaments are made up of bundles of thin threads or subfilaments.
One of the most magnificient elephant trunks in the Rosette Nebula is shown in the lower half of Fig. 1. (position: $6^{\mathrm{h}} 31^{\mathrm{m}} 34^{\mathrm{s}}+5^{\circ} 9^{\prime}$, epoch: 2000.0). The trunk consists of an elongated structure of the size $\sim 55^{\prime \prime} \times 260^{\prime \prime}$, corresponding to $\sim 0.37 \mathrm{pc} \times 1.75 \mathrm{pc}$ in projected measures at the adopted distance. To a certain degree the trunk resembles a wrench with its two jaws. Hence, we shall denote it the Wrench Trunk. The trunk presents a wealth of structures and details, primarily in the form of dark filaments, subfilaments, and knots. In fact, the whole trunk seems to be built up by such elements. The 


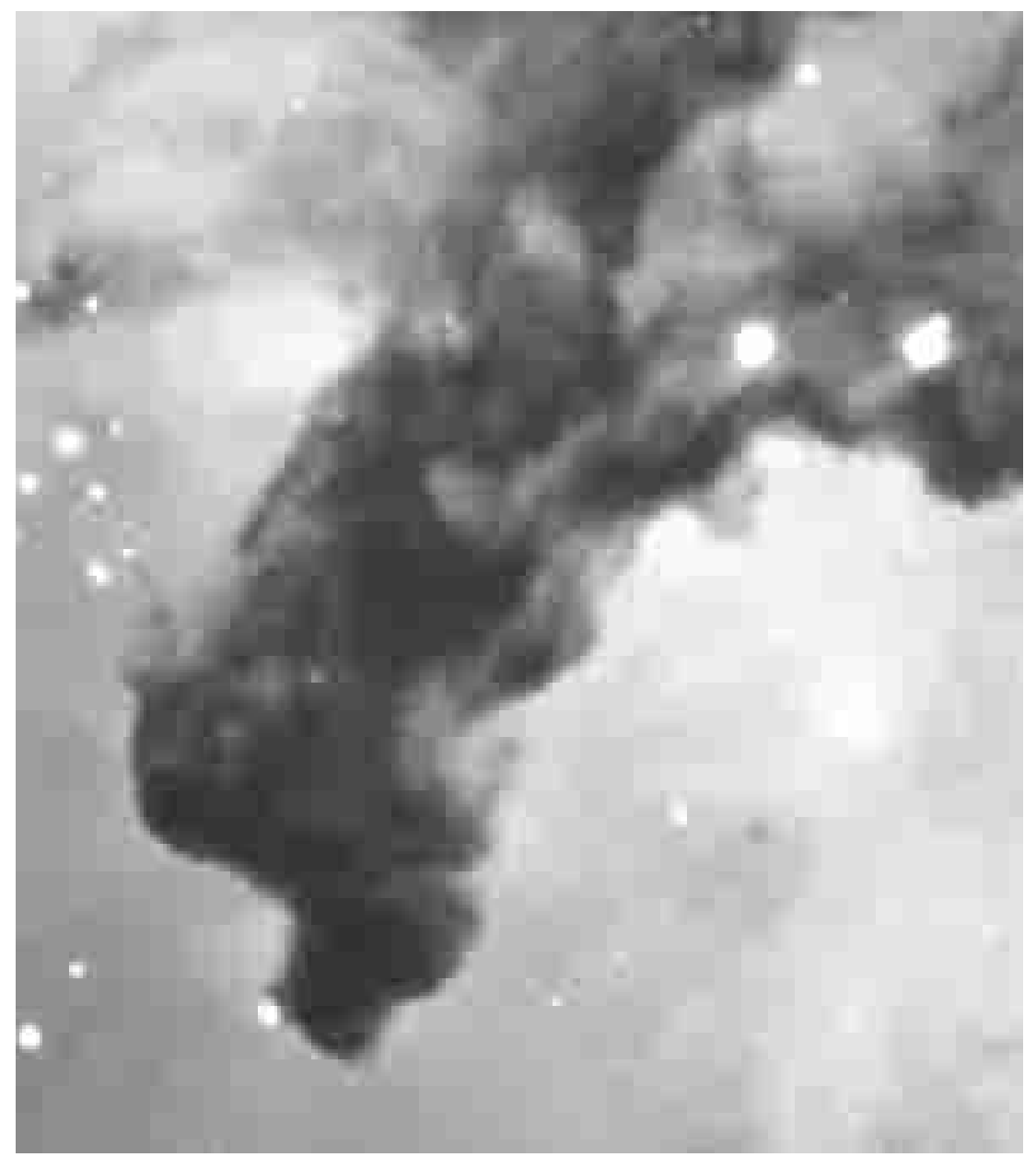

Fig. 2. Enlargement of one of the elephant trunks in Fig. 1. Two pronounced, serpentine-shaped legs are running from the north and from the west and combine in the head of the trunk. The serpentines are found to consist of a number of dark subfilaments. The image covers $84^{\prime \prime} \times 94^{\prime \prime}$.

thickness of the subfilaments are mostly in the range $\sim 1^{\prime \prime}-4^{\prime \prime}$, corresponding to $\sim 1400-5600$ au. Many of them have a wavy shape, and some are organized in bundles.

A conspicuous feature of the trunk is that the filaments and subfilaments appear to be twisted into a helical geometry. This is particularly evident in the upper two thirds of the trunk where they can be most readily followed. In the lower part of the trunk there are two jaws which appear to have an internal filamentary structure which may be twisted. At the tip of each of the jaws a globule is about to break off. A few globules have already been released from the eastern jaw. At its northern end the trunk is split into two bundles of subfilaments running mainly in the north-east and west-north-west directions.

Above, and slightly east of the Wrench Trunk, close to the centre of Fig. 1, there are two trunks with a bay in between. In Fig. 2 we show an enlarged and more detailed view of the eastern one of these trunks $\left(6^{\mathrm{h}} 31^{\mathrm{m}} 39^{\mathrm{s}}+5^{\circ} 12^{\prime}\right)$. The thickness of the trunk is $\sim 30^{\prime \prime}$ corresponding to $\sim 0.20 \mathrm{pc}$. Also this trunk consists of a large number of dark filaments and subfilaments. Particularly striking is the vigorous corkscrew-shaped filament penetrating the trunk from the north-north-west. Another meandering filament comes from the west, turns more to the south, and appears to amalgamate with the previously mentioned filament just above the head of the trunk. Both filaments consist of a number of subfilaments which in some places appear to wind around one another. In the head of the trunk there is also a filamentary substructure, mainly running in the toroidal direction.

IC 1805. Another elephant trunk with two jaws is shown in Fig. $3\left(2^{\mathrm{h}} 36^{\mathrm{m}} 35^{\mathrm{s}}+61^{\circ} 23^{\prime}\right)$. It is situated in the H II region IC 1805 at an estimated distance of $2.35 \mathrm{kpc}$ (Massey et al. 1995) and bears some remblance to a stag-beetle. The projected size of the trunk is $\sim 80^{\prime \prime} \times 190^{\prime \prime}$, corresponding to $\sim 0.9 \mathrm{pc} \times 2.2 \mathrm{pc}$. The trunk consists of a great number of winding dark filaments and subfilaments which mainly run diagonally across the trunk, indicating a twisted structure of the trunk. The head of the trunk (in the north-west) is sustaining two principal jaws that both appear to have a twisted structure. A number of smaller outgrowths can also be seen in connection with the southern jaw. In the northern jaw at least two subfilaments are twined to a winding unity. The two principal jaws are probably of the same nature as the jaws in the Wrench Trunk in the Rosette Nebula (see also Sect. 6). At its base the trunk is split up in two bundles of filaments which are mainly running southwards and eastwards.

To the south of the main trunk in Fig. $3\left(2^{\mathrm{h}} 36^{\mathrm{m}} 32^{\mathrm{s}}+61^{\circ} 21^{\prime}\right)$ there are a number of smaller and less developed trunks, all pointing in the same direction as the main trunk. An enlargement of this portion of the figure is shown in Fig. 4. The figure 


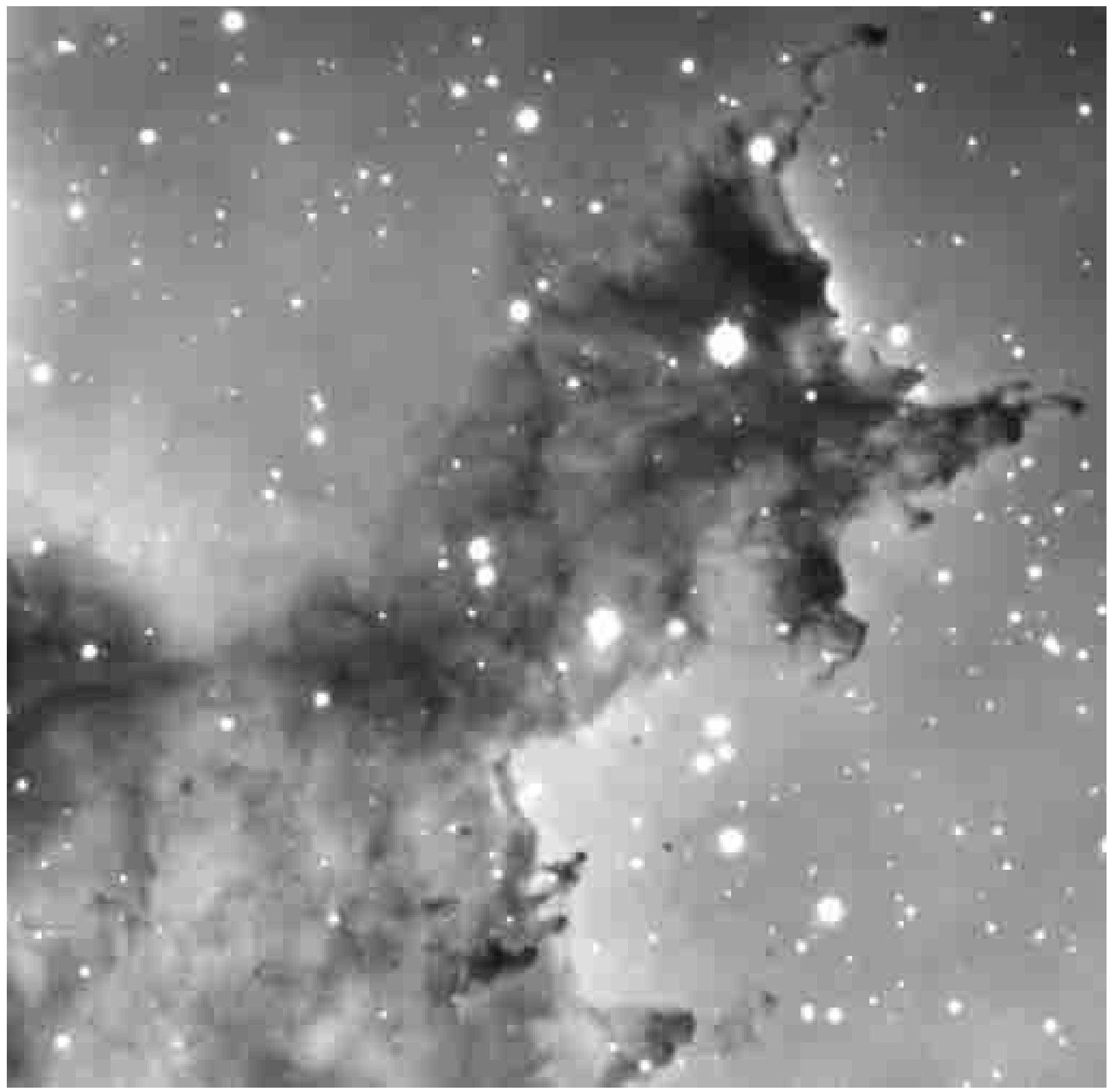

Fig. 3. One of the most prominent elephant trunks in IC 1805. The trunk is built up by a number of dark filaments, many of which are running diagonally across the trunk indicating a twisted structure. South of the big trunk there are several smaller trunks of simpler shape. Image size, $265^{\prime \prime} \times 260^{\prime \prime}$. North is up.

reveals that most of the trunks are built up by a comparatively dense head and two legs of V-shape. The head of the largest of these trunks is distinctly double. In principle this could mean that there are either two separate trunks with two separate heads seen in projected superposition, or there is a single trunk with its head split in two parts, perhaps resembling the jaws in the Wrench Trunk. The question may be solved by considering the connecting legs which seem to consist of twined subfilaments that are connected with both of the heads. For this reason we find the latter alternative to be the more likely one. We also want to pay attention to the fact that there are at least two more trunks in the image that show signs of a split head and V-shaped legs.

$N 81, S M C$. Finally we shall consider a trunk which is much more distant than the trunks discussed above. We have chosen the main trunk $\left(1^{\mathrm{h}} 9^{\mathrm{m}} 12^{\mathrm{s}} \cdot 6-73^{\circ} 11^{\prime} 41^{\prime \prime}\right)$ in the H II region $\mathrm{N} 81$ in the SMC which has been studied by Heydari-Malayeri et al. (1999) using the Hubble Space Telescope (HST). Figure 5 shows part of the HII region with the trunk near the centre. The H II region is excited by radiation, winds, and shocks originating from a small cluster of very young and massive stars. A molecular cloud seems to be associated with $\mathrm{N} 81$ 


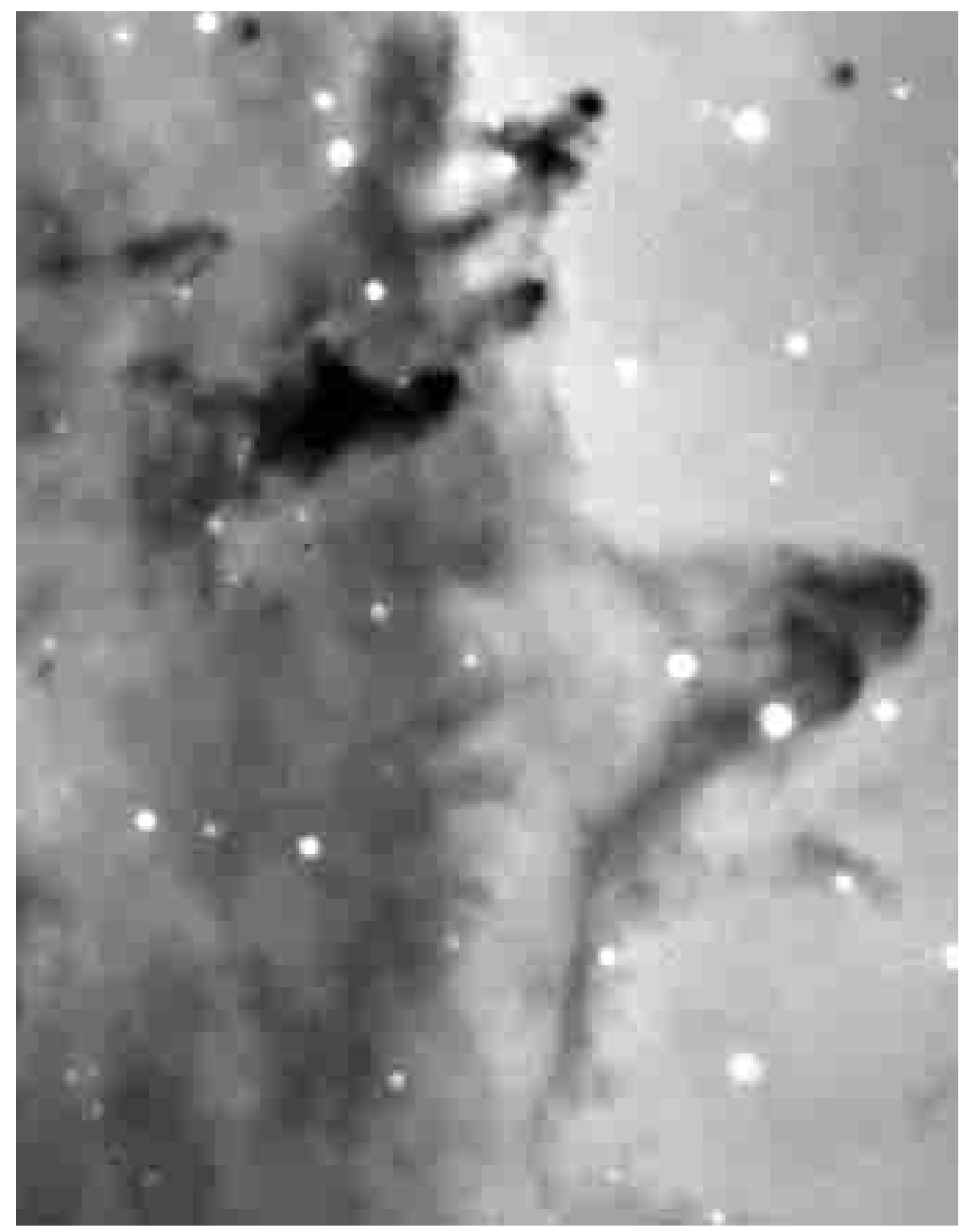

Fig. 4. Enlargement of the southern part of Fig. 3 showing some of the smaller elephant trunks in IC 1805. Most of the trunks are intrinsically filamentary and all are connected by filamentary legs to the dark background eastwards. Image size, $78^{\prime \prime} \times 100^{\prime \prime}$.

(Israel et al. 1993). As is evident from Fig. 5 the dark structures are essentially Y-shaped. The lower part of the Y constitutes the very elephant trunk with a dark blob forming its head. Smoothly connected with the upper part of the trunk are two legs which make up the rest of the Y. Each of the legs seems to consist of two or more filaments. The projected length of the trunk is 1 ".6 corresponding to $0.5 \mathrm{pc}$ at an adopted distance of $63 \mathrm{kpc}$ (e.g. Di Benedetto 1997, and references therein). One may ask whether the trunk is twisted or not. Unfortunately, the angular resolution of even the HST is in this case not good enough to definitely settle the question. We note, however, that the trunk shows a knotty structure indicating that it really is twisted.

From the examples of elephant trunks given above we may draw the following conclusions. 1) The trunks either are fairly well-developed with a complex fine structure, or there is only a dark blob. 2) All well-developed trunks are made up of dark filaments, subfilaments and knots instead of being featureless bodies. 3) In the well-developed trunks there is evidence of twisted structures. 4) The trunks are connected with essentially two filamentary legs which run in V-shape from the trunks. Often the legs consist of bundles of filaments. The angle of the V may vary. 5) In all trunks the extinction is maximum in their heads. The shapes and properties discussed here are also widespread in the larger sample of elephant trunks we have observed.

\section{A mechanical analogy model}

In CGK02 we described a mechanical analogy model which may shed some light on the physical background of the double helix mechanism being so vital to our theory. In the following we shall develop the analogy model a little bit further and give an account of some experiments performed. Since long 


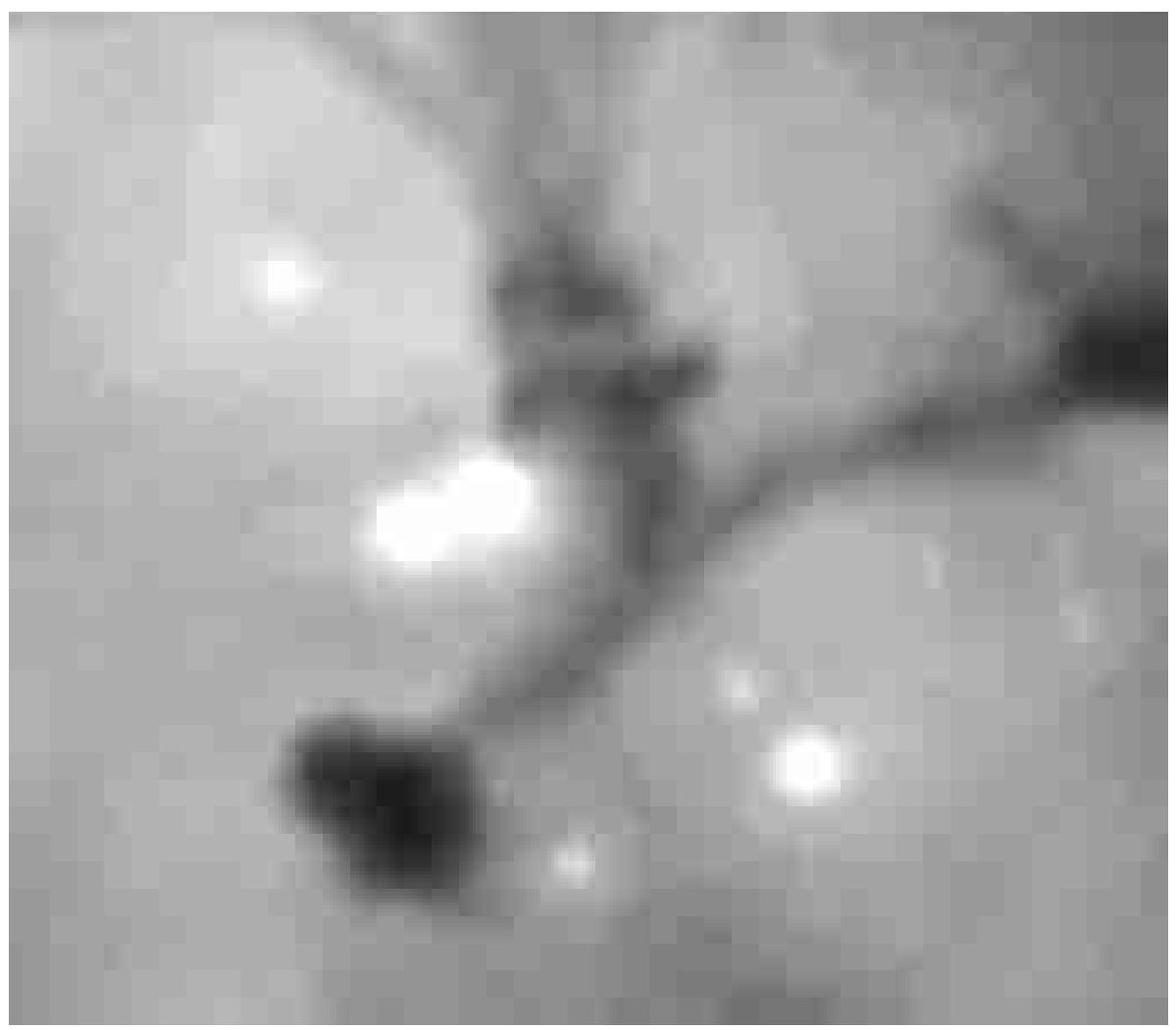

Fig. 5. Elephant trunk in the central part of N 81 in the SMC (Credit: NASA and The Hubble Heritage Team (STScI/AURA). For a complete image of $\mathrm{N} \mathrm{81}$, see Heydari-Malayeri et al. 1999). The trunk is made up of a pronounced head connected to a dark pillar which possibly consists of intertwining filaments. The pillar is split into two filamentary legs so that the whole dark structure resembles a Y. The image covers $3 " .9 \times 3 "$." South-west is up.

it has been well-known that magnetic field lines can, in many respects, be compared to elastic strings (Faraday 1839-1855; Alfvén 1950). On this basis we have constructed an analogy model to illuminate some of the properties of our trunk model. In this model a bundle of elastic strings is stretched between two fixed positions, $\mathrm{A}$ and $\mathrm{C}$, situated at the same horizontal level. The bundle represents the magnetic flux tube in the trunk model, while the separate strings imitate the magnetic field lines. Straight strings are thus equivalent to straight magnetic field lines implying that there is no axial electric current present. If we twist the bundle of strings at one of its ends, say A, the strings become helically shaped. This is equivalent to helically shaped magnetic field lines, and to a finite axial electric current in the flux tube. The bundle of strings studied thus constitutes a mechanical analogue of a magnetic flux tube.

We now load the bundle of strings half-way between A and $\mathrm{C}$ with a weight of mass $m$ which is acted upon by the gravitational field. This imitates the mass condensation in the filament with its excess inertia force. If the individual elastic strings in the bundle are initially straight the bundle will hang down in a V-shape with the weight at its apex.

If instead the bundle of strings is twisted at one end through an angle $\phi_{0}$, one out of two fundamentally different geometries may result. If on the one hand the twist angle $\phi_{0}$ is sufficiently small the bundle remains V-shaped. If on the other hand $\phi_{0}$ exceeds a certain critical value, $\phi_{0 \mathrm{c}}$, the bundle forms a double helix above the weight (Fig. 6). It is found that the double helix

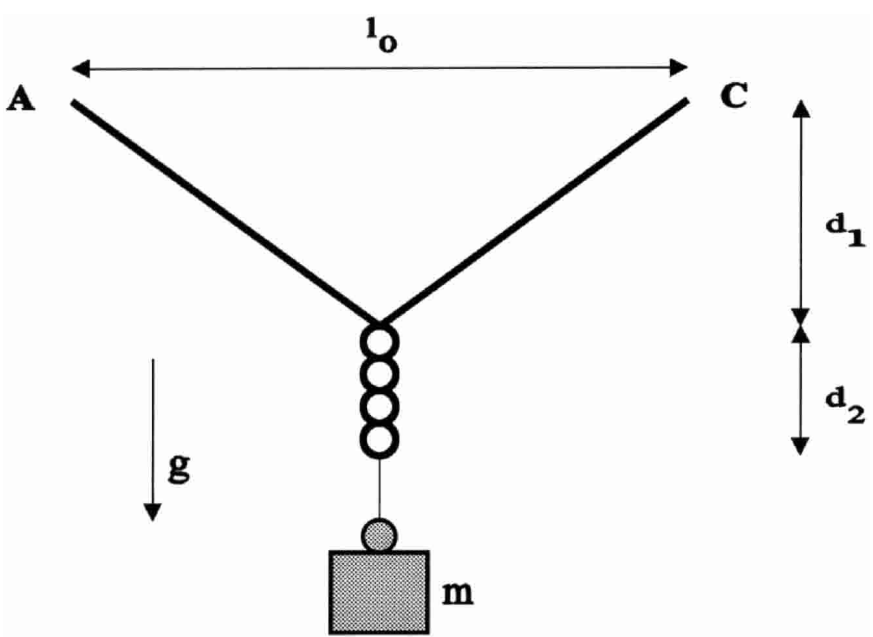

Fig. 6. Schematic picture of the mechanical analogy model. A bundle of elastic strings is loaded by a weight of mass $m$. If the bundle is twisted a sufficient number of turns it will form a double helix just above the weight.

and the bundle of strings are twisted in opposite ways. From the upper end of the double helix the bundle runs in $\mathrm{V}$-shape towards $\mathrm{A}$ and $\mathrm{C}$, so that the whole structure of the bundle takes the shape of a Y. This configuration has many features in common with the Wrench Trunk and its connecting filaments (Fig. 1). 


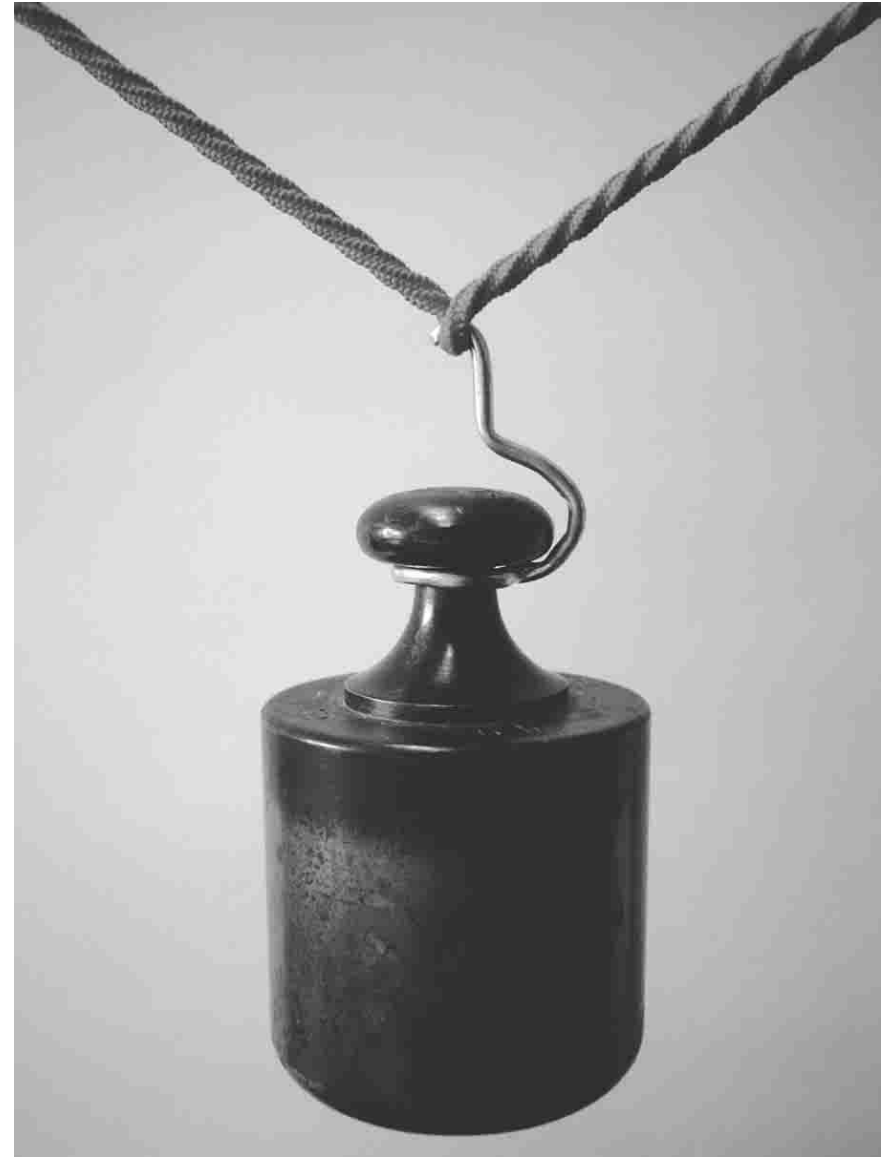

Fig. 7. Detail of the analogy model comprising a loaded elastic string. The string is twisted just enough for a double helix to start developing. The string consists of five thin substrings, which give rise to the grooves on its surface, reflecting the geometry of the magnetic field lines inside theflux tube. It is to be noticed that the double helix and the string are twisted in opposite ways.

We have performed a number of experiments with the analogy model for various combinations of the initial tensional force and mass of the weight. The bundle consisted of five rubber strings. Among other things we measured the height of the V-shaped portion, $d_{1}$, and the length of the double helix, $d_{2}$, as functions of the twist angle $\phi_{0}$. In one set of such experiments the bundle had a length $l_{0}=0.52 \mathrm{~m}$ and a radius $R_{0} \approx 1.5 \times 10^{-3} \mathrm{~m}$. The applied weight was $m=0.5 \mathrm{~kg}$ yielding the force $m g=4.91 \mathrm{~N}$. The results of the experiments are shown in Fig. 8. For small values of $\phi_{0}$ the height $d_{1}$ increases slowly with $\phi_{0}$ while $d_{2}$ is zero. In these cases the bundle of strings has a V-shape only. When $\phi_{0}$ reaches the critical value $\phi_{0 \mathrm{c}} \approx 23.7$ turns $(\approx 149 \mathrm{rad})$ the first sign of a double helix appears (Fig. 7). For increasing values of $\phi_{0}$ the double helix becomes more and more developed with an increasing length, $d_{2}$, and an increasing number of turns. At the same time $d_{1}$ decreases. It is to be noted that the sense of twist observed in the double helix is opposite to the sense of twist in the bundle of strings.

\section{A theoretical model of the double helix mechanism}

\subsection{Basic properties of the model}

In order to further illustrate the physics behind the formation of the double helix we shall consider a simple model where a magnetic flux tube containing a conducting gas or fluid is locally influenced by an external force. The magnetic Reynolds number is supposed to be large so that the magnetic field is well frozen in to the fluid. We assume that the flux tube initially takes the form of a cylinder of radius $R_{0}$ running between the two positions $\mathrm{A}$ and $\mathrm{C}$ which are separated by the distance $l_{0}$. Cylindrical coordinates $(r, \phi, z)$ are adopted with the $z$-axis coinciding with the axis of the cylinder. The magnetic field $\boldsymbol{B}$ has a $z$-component that is assumed to be homogeneous, $B_{z}=B_{0}$. If one end of the tube is twisted through the angle $\phi_{0}$ a toroidal magnetic-field component of strength $B_{\phi}=\phi_{0} r B_{0} / l_{0}$ is also generated leading to helical magnetic field lines. For $r=0$ the magnetic field is aligned with the axis of the flux tube, while for larger values of $r$ the helix angle increases with $r$.

Half-way between $\mathrm{A}$ and $\mathrm{C}$ a force $\boldsymbol{F}$ is applied perpendicularly to the magnetic flux tube. The force corresponds to the excess inertia force mentioned in Sect. 2. It is of obvious interest to find out the shape of the flux tube in the case the applied force $\boldsymbol{F}$ is in equilibrium with the magnetic tensional force. If the magnetic field lines are only axially directed in the flux tube, i.e. $\phi_{0}=0$, the tube will adopt the shape of a V. Also for sufficiently small values of the twist angle $\phi_{0}$ the flux tube is expected to be of V-shape only, with no double helix present. If the angle at the apex of the $\mathrm{V}$ is denoted by $2 \beta$, the total length of the flux tube is $l=l_{0} / \sin \beta$. Assuming a constant volume of the flux tube we obtain the new tube radius $R=R_{0} \sin ^{1 / 2} \beta$. Conservation of the magnetic flux in the tube leads to an axial component of the magnetic field $B_{z}=B_{0} / \sin \beta$. Using the twist angle, $\phi_{0}$, we find the toroidal component of the magnetic field in the flux tube to be $B_{\phi}=\phi_{0} B_{0} r / l_{0}$. The magnetic energy per unit length of the flux tube can then be expressed as

$w_{B}=\frac{\pi R_{0}^{2} B_{0}^{2}}{2 \mu_{0}}\left(\frac{1}{\sin \beta}+\frac{\phi_{0}^{2} R_{0}^{2} \sin ^{2} \beta}{2 l_{0}^{2}}\right)$.

Integrating Maxwell's stresses

$\boldsymbol{T}=\frac{\boldsymbol{B}(\boldsymbol{n} \cdot \boldsymbol{B})}{\mu_{0}}-\frac{B^{2} \boldsymbol{n}}{2 \mu_{0}}$

over a thin cross-sectional volume of the tube in the symmetry plane through the apex of the $\mathrm{V}$, we obtain the total tensional force opposing the applied force

$F_{B}=\frac{2 \pi R_{0}^{2} B_{0}^{2} \cos \beta}{\mu_{0}}\left\{\frac{1}{\sin \beta}-\left(\frac{\phi_{0} R_{0} \sin \beta}{2 l_{0}}\right)^{2}\right\}$.

In equilibrium we have $F_{B}=F$. If there is no twist of the field lines, $\phi_{0}=0$, we find from (3), $\cot \beta=\mu_{0} F /\left(2 \pi R_{0}^{2} B_{0}^{2}\right)$, yielding the height of the $\mathrm{V}$-shaped filament

$d_{1}=\frac{\mu_{0} l_{0} F}{4 \pi R_{0}^{2} B_{0}^{2}}$. 


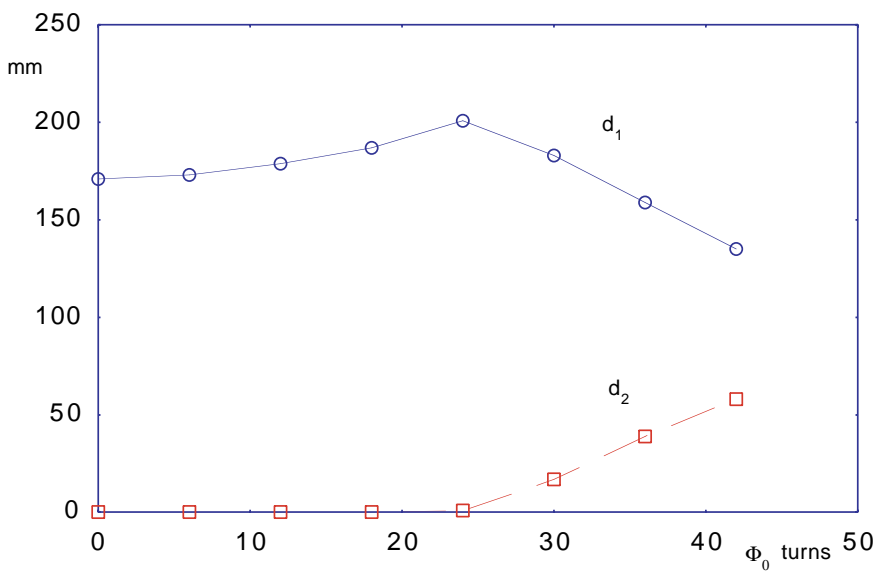

Fig. 8. Results of experiments with the mechanical analogy model. The distances $d_{1}$ and $d_{2}$ (for definitions see Fig. 6) are plotted as functions of the twist angle $\phi_{0}$. For $\phi_{0}<\phi_{0 \mathrm{c}} \approx 23.7$ turns the bundle of strings adopts a V-shape. For $\phi_{0}>\phi_{0 \mathrm{c}}$ the bundle of strings also forms a double helix.

How can we now find out under what conditions a double helix is formed in the flux tube system? To solve this problem we shall make use of two important principles. The first principle states that the system tends towards a state where the total, static energy is minimum. The second principle is based on the fact that the helicity of the magnetic field $H=\langle\boldsymbol{A} \cdot \boldsymbol{B}\rangle$ is conserved since the magnetic Reynolds number is large (Woltjer 1958a; Moffatt 1969). Here, $\boldsymbol{A}$ is the vector potential for the magnetic field $\boldsymbol{B}$, while $<\cdot>$ stands for a volume average over the flux tube.

The magnetic field in our system may, under certain conditions, be considered to be twisted on two different levels. Firstly, if $\phi_{0} \neq 0$, the magnetic field is twisted inside the flux tube. Secondly, part of the flux tube, with its inherent magnetic field, may be twisted into a double helix. Each of these two modes of twisting contributes to the helicity (Moffatt \& Proctor 1985; Moffatt \& Ricca 1992, and references therein). Since the helicity has to be constant, a change of one of the contributions will necessarily lead to a change of the other contribution with the same amount but of opposite sign. The constancy of the helicity implies that if a flux tube is twisted into a double helix of $M$ turns, the number of turns of the magnetic field lines inside the flux tube will be changed by an amount of $2 M$ turns (Alfvén 1950; Moffatt \& Proctor 1985). The change is negative if the sense of twist of the double helix is opposite to the sense of twist of the magnetic field lines inside the filament. Otherwise it is positive.

\subsection{A numerical model}

In order for a filament to form a double helix, the energy state of the filament including the double helix has to be lower than the energy state of a corresponding, simple V-shaped filament in force equilibrium. To find out the conditions under which this is valid, we have developed a code that solves the problem numerically. Our model filament is presumed to be initially twisted through the angle $\phi_{0}$. To begin with we assume the

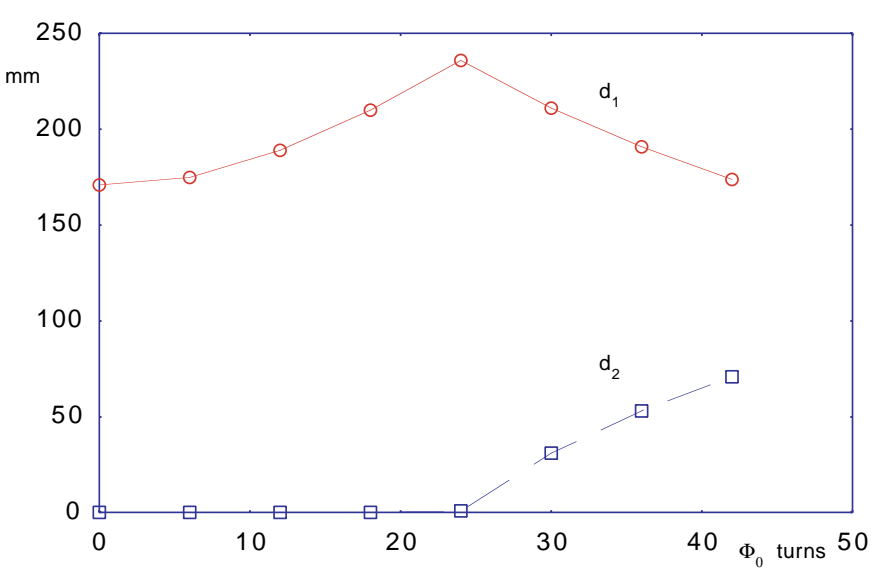

Fig. 9. Results of numerical calculations using the theoretical model. The distances $d_{1}$ and $d_{2}$ are plotted as functions of the twist angle $\phi_{0}$. The values of $l_{0}$ and $F$ are identical to those of the analogy model, and the values of $R_{0}$ and $B_{0}$ have been adapted so that $d_{1}\left(\phi_{0}=0\right)$ and $\phi_{0 c}$ are the same as in the experiments. The similarity of the shapes of the curves in Figs. 8 and 9 is obvious.

filament to be V-shaped as a result of the action of the applied force $\mathrm{F}$, and calculate its height, $d_{1}$, using (3) and $F_{B}=F$. We then introduce a deformation of the filament at the apex of the $\mathrm{V}$ in the form of a double helix with a tiny twist (a small fraction of a turn), and calculate the change of the total, static energy of the system, including the magnetic energy and the potential energy of the applied force. If the change of the total energy is positive there is no tendency towards the formation of a double helix, and hence the filament will remain V-shaped.

If the change of the static energy is negative, a lower state of this energy exists implying the presence of a double helix. A new, small increment is then added to the twist of the double helix, and new calculations of $d_{1}, l, R$, and $B$, and the length of the double helix, $d_{2}$, are performed. For the calculations we have used a tensional force of the filamentary magnetic field given by (3), and a magnetic energy density per unit length of the filament given by (1). These equations are strictly valid only for the straight parts of the filament, but constitute a firstorder approximations for the double helix where the filament is curved. A new test of the sign of the change of the static energy is subsequently done for the new set of parametres. This procedure is repeated until the change of the static energy is positive, indicating that the minimum of the static energy has been reached. The state found represents the final equilibrium of the filamentary system. The calculations yield, among other things, values of the parametres $d_{1}, d_{2}, l, R, B$, and the number of turns, $\theta$, of the double helix.

\subsection{Comparison with the analogy experiments}

We shall now compare the results of the analogy experiments discussed in Sect. 4 and the results of the numerical model described in the previous section. In order to make the comparison as convenient as possible we have adopted the same values of $l_{0}$ and $F$ as those chosen in the experiments. Using the model we have adapted $R_{0}$ and $B_{0}$ so that the values of the two quantities $d_{1}\left(\phi_{0}=0\right)$ and $\phi_{0 \mathrm{c}}$ are the same as those in the 
experiments. This procedure results in a value of $R_{0}$ that is about four times larger than the radius of the string in the experiments. The discrepancy is mainly due to the fact that the filamentary magnetic field has not the same tension-stretching characteristics as the rubber string used. With the adopted values of $l_{0}, R_{0}, F$, and $B_{0}$ we have calculated $d_{1}$ and $d_{1}$ for various values of $\phi_{0}$. The result is shown in Fig. 9. It is clear from the figure that $d_{2}=0$ for twist angles in the interval $0<\phi_{0} \leq \phi_{0 \mathrm{c}} \approx 23.7$ turns $(\approx 149 \mathrm{rad})$. No double helix exists in this interval, and the filament forms a V only. The height of the $\mathrm{V}$ is found to increase somewhat with increasing $\phi_{0}$ up to $\phi_{0 \mathrm{c}}$. For $\phi_{0}>\phi_{0 \mathrm{c}}$ a double helix appears from the apex of the V. Just as in the experiment the helix is twisted in an opposite way as compared with the interior of the filament. In the $\phi_{0}$-regime considered $d_{2}$ increases nearly linearly with $\phi_{0}$ while $d_{1}$ decreases. A comparison of Figs. 8 and 9 shows that the similarity between the experimental results and the results computed from the model is striking. Both $d_{1}$ and $d_{2}$ are found to vary with $\phi_{0}$ in essentially the same way. Only some minor deviations exist.

In the model discussed above we have assumed that the filament has a constant volume. This is suitable when comparing the results of the model with the rubber string experiments since rubber, when deformed, maintains its volume fairly well. As regards our trunk theory we do not yet know with any certainty which relation that exists between the radius and the length of the magnetic flux tube. It is therefore appropriate to ask whether a double helix may form also under other conditions. Numerical calculations performed show that a double helix can indeed also form for other relations between the radius and the length.

\section{Application of the theory to the Wrench Trunk}

The Wrench Trunk represents something of an archetype of our trunk model. It is therefore of obvious interest to apply the theory to it. In this matter we need to know the values of the relevant parameters $l_{0}, R, B_{1}$, and $\alpha$. Several, but not all, of these quantities can be found from the image of the trunk (Fig. 1) and the adopted distance. We thus find that the present radius of the filament is $\sim 15^{\prime \prime}$, which corresponds to $R \approx 3.1 \times 10^{15} \mathrm{~m}$. We also estimate that the trunk is twisted by $\theta \approx 2-3$ turns. The axis of the trunk is most likely not perpendicular to the line of sight. Schneps et al. (1980) assumed the tilt angle to be $\psi=45^{\circ}$ with the head of the trunk tilted away from us. In the following we shall stick to this value. The angular distance between the northern ends of the connecting bundles of filaments, corresponding to the positions $\mathrm{A}$ and $\mathrm{C}$ in Fig. 6, is estimated to be $\sim 400^{\prime \prime}$ leading to $l_{0} \approx 8 \times 10^{16} \mathrm{~m}$. The apparent angle between the upper legs of the $\mathrm{Y}$ is measured to be $2 \beta_{\text {app }} \approx 105^{\circ}$ with an uncertainty of a few degrees. With the assumed tilt of the trunk, $\psi=45^{\circ}$, the true angle of the $\mathrm{Y}$ is $2 \beta \approx 85^{\circ}$. Again, using $\psi=45^{\circ}$ and the adopted distance we obtain $d_{1} \approx 4 \times 10^{16} \mathrm{~m} \approx 1.3 \mathrm{pc}$ and a true length of the trunk $d_{2} \approx 8 \times 10^{16} \mathrm{~m} \approx 2.5 \mathrm{pc}$.

Applying the constant-volume model to the Wrench Trunk while putting $l_{0} \approx 8 \times 10^{16} \mathrm{~m}$ we aim at $2 \beta=85^{\circ}$ and $\theta=2.5$ turns by choosing an appropriate set of values of $B_{0}, F, R_{0}$, and $\phi_{0}$. Numerical calculations show that one such set is $B_{0} \approx 2 \times 10^{-9} \mathrm{~T}(=20 \mu \mathrm{G}), F \approx 1.2 \times 10^{21} \mathrm{~N}, R_{0} \approx 5.8 \times 10^{15} \mathrm{~m}$ and $\phi_{0} \approx 17.8$ turns. Here we have chosen $20 \mu \mathrm{G}$ as a reasonable value of $B_{0}$. However, also other sets with the same value of $R_{0}$ and $\phi_{0}$, and with $B_{0}$ and $F$ fulfilling $B_{0}{ }^{2} / F \approx 3.3 \times 10^{-39}$ $\mathrm{T}^{2} / \mathrm{N}$, are capable of simulating the Wrench Trunk. The geometry of the flux tube remains the same for all such sets.

With the set of values of $B_{0}, R_{0}, F$, and $\phi_{0}$ given above we obtain the axial component of the present magnetic field in the tube, $B_{z} \approx 6.6 \times 10^{-9} \mathrm{~T}(=66 \mu \mathrm{G})$. The total electric current in the magnetic flux tube consistent with this case is $\sim 1 \times 10^{14} \mathrm{~A}$. For a comparison, we may recall that this current is only about two orders of magnitude larger than typical currents in large solar prominences. The current found corresponds to a mean current density of $\sim 3 \times 10^{-18} \mathrm{~A} \mathrm{~m}^{-2}$. Assuming a density of the $\mathrm{H}_{2}$ gas in the trunk of say, $5 \times 10^{4} \mathrm{~cm}^{-3}=5 \times 10^{10} \mathrm{~m}^{-3}$, and a degree of ionization of $10^{-7}$ we find that the systematic speed of the current carrying particles is only $\sim 0.004 \mathrm{~m} \mathrm{~s}^{-1}$. This speed is by far too small to give rise to any appreciable ohmic heating of the molecular gas in the flux tube.

Schneps et al. (1980) have observed the Wrench Trunk in ${ }^{12} \mathrm{CO}$ and ${ }^{13} \mathrm{CO}$, and have among other things studied the mass distribution in the trunk. They find that the mass is very inhomogeneously distributed along the trunk. The dominant part of the mass, $\sim 10 M_{\odot}$, is concentrated to the head of the trunk while the rest of the trunk contains less than $7 M_{\odot}$. A qualitatively similar conclusion may also be drawn from Fig. 1 which reveals that the extinction is maximum in the head of the trunk whereas the middle and northern parts are more dilute. From their CO data Schneps et al. also determined the distribution of the radial velocity in the trunk. They found a fairly regular radial velocity gradient of about $1 \mathrm{~km} \mathrm{~s}^{-1} \operatorname{arcmin}^{-1}$ along the axis of the trunk corresponding to a stretching of the trunk of $\sim 5 \mathrm{~km} \mathrm{~s}^{-1}$.

Using the inertia force $F \approx 1.2 \times 10^{21} \mathrm{~N}$ obtained above and the estimated mass of the head of the trunk $\sim 10 M_{\odot} \approx 2 \times$ $10^{31} \mathrm{~kg}$ we find that the present acceleration of the head caused by magnetic tensional forces is $\sim 6 \times 10^{-11} \mathrm{~m} \mathrm{~s}^{-2}$. Schneps et al. estimated the age of the trunks to about $5 \times 10^{5}$ years $\approx$ $1.5 \times 10^{13} \mathrm{~s}$. Since the mean acceleration due to magnetic tension during the lifetime of the trunk is expected to be less than half of the present acceleration the change of the speed of the head should be less than $0.5 \mathrm{~km} \mathrm{~s}^{-1}$. This is negligible compared with the stretching. The head of the trunk is also acted upon by the radiation pressure and gravity of NGC 2244, the forces of which are presently comparable the magnetic tensional force.

A prominent feature of the Wrench Trunk is constituted by the two jaws which clearly stand out from the head. We interpret these jaws to be the remnants of the dark filamentary loop that once closed the double helix in its southern part. Here, the radiation from NGC 2244 is maximum, and here the erosion is also expected to be maximum. It is to be noticed that even if a considerable fraction of the initially dark filaments in the loop are not visible any more, the magnetic field has still to be closed and form a loop.

Jaw-like structures of similar kind are found also in other elephant trunks. One example of this is the pair of jaws in 
the Stag-Beetle Trunk in IC 1805 (Fig. 3). Here we see that, in particular, the southern jaw has a number of small outgrowths. In our scheme these outgrowths may be interpreted as the visible remains of the densest filaments in the flux tube which have best resisted the radiation from the central hot stars.

When discussing the outgrowths of the jaws of the StagBeetle Trunk the thoughts are involuntarily carried to the so called EGGs (Evaporating Gaseous Globules) that were discovered a few years ago by Hester et al. (1996a) at the surface of the now so famous elephant trunks in the Eagle Nebula. They hypothesised that the EGGs were formed as the result of a shadowing effect of very young stars surrounded by dense cocoons of gas. The dense cocoons should be eroded less quickly than the rest of the dark cloud and should hence stand out as EGGs. A number of authors have later studied the Eagle Trunks in various bands reaching from near-infrared to mmwavelengths, and found only few indications of young stellar objects (McCaughrean 1997; Pilbratt et al. 1998; White et al. 1999). Recently McCaughrean \& Andersen (2002) have observed the trunks in the near-infrared with the Very Large Telescope, and found that most of the EGGs appear to be empty of stars. Only some $15 \%$ of them may house low-mass stars or brown dwarfs. Here, we want to pay attention to the idea that the EGGs may be of the same nature as the outgrowths in e.g. the Stag-Beetle Trunk. According to this view, most of the EGGs should be the remnants of the densest filaments in the trunks, i.e. the ones which have best withstanded the eroding radiation of the central hot stars. This does not exclude that star formation may take place in some of these filaments.

\section{Discussion}

In the elephant trunk model discussed above the magnetic field has a profound impact on the shape of the trunks and the filaments out of which they are made. According to the model the magnetic field may be twisted on two different levels. On the first level the magnetic field lines inside the flux tube are twisted into helical shapes by external forces. On the second level the very flux tube is twisted into a double helix. The double-helix formation may therefore represent a kind of selforganization which takes place as a result of the feeding of energy into the system.

The model of the double helix mechanism treats a steady situation where all forces are in equilibrium. Even if the model is capable of accounting for many of the basic features observed in elephant trunks, it can only be considered a first approximation. The reason for this is that the force applied to the magnetic flux tube in the model corresponds to an inertia force in the trunk system. This implies that the trunk must be accelerated, and hence cannot be steady. To describe the development properly, a dynamical model is required. Under certain conditions, however, the steady state model may give a reasonably good description also of the dynamical system. If the time-development of the double helix with its V-shaped legs is sufficiently slow, one may consider the development as a series of stationary states following one another. For this to be the case, it is required that the stretching of the V-shape and the twisting of the double helix involve energies that are small compared with other energies invoved in the process.

The process leading to the formation of a double helix in a mass loaded magnetic flux tube, has some features in common with the formation of loops in a straight magnetic flux tube discussed by Alfvén long ago (Alfvén 1950; Alfvén \& Fälthammar 1963). In fact, the situation treated by Alfvén may be interpreted as a special case of our mass loaded flux tube, viz. when the applied force $F$ in our model is reduced to zero. Using a very small applied force, so that $2 \beta$ is close to $180^{\circ}$, we find from our numerical model that the helix angle is $\theta \approx 51^{\circ} .0$ when the double helix is just about to form. Alfvén found that the corresponding helix angle in his special case is arctan $(\sqrt{5}-1) \approx 51^{\circ}$.03. Alfvén interpreted his loop formation as an example of the kink-instability. Why then, did Alfvén classify his loop formation as an instability while we have argued that our helix represents a stable structure? The solution to this apparent contradiction is connected with how the electric current in the flux tube is generated. If, on the one hand, the magnetic flux tube is of finite length and twisted a certain number of turns at one of its ends, as we assume, there will be a stable situation. The reason for this is that the helix angle will become reduced as soon as the double helix (or loop) starts to form. If, on the other hand, the magnetic flux tube is infinitely long or there is a constant-current device connected to the tube, the helix angle will remain constant and, hence, the formation of a double helix (or loops) may go on indefinitely.

Another instability that might be of interest in this connection is the Kruskal-Shafranov (K-S) instability, which may also develop into a kink (e.g. Kadomtsev 1966). This instability occurs for filamentary currents that are considerably smaller than those required for the Alfvén instability, and corresponds to twists of the magnetic field which may be as small as one turn along the filament. When comparing these two instabilities we note that many of our filaments appear to be twisted much more than one turn (see e.g. Fig. 2). Thus, there is an apparent problem in combining these structures with the presence of an active $\mathrm{K}-\mathrm{S}$ instability.

What may then be the reason for the shortcoming of the K-S instability? Kruskal and Shafranov aimed primarily at applications to fusion plasma devices where the current-carrying plasma filament is surrounded almost entirely by vacuum. The $\mathrm{K}-\mathrm{S}$ instability is based on the fact that the magnetic energy outside the current filament is larger in the initial, undisturbed state than when the instability has started to develop (Kadomtsev 1992). Hence, the external magnetic field feeds the instability. A completely different situation prevails for the flux tubes considered here in connection with the twisted elephant trunks. These tubes contain a cold and dusty plasma surrounded by a hot plasma of high conductivity and frozen-in magnetic fields. The external plasma can therefore be considered magnetically screened from the flux tube. As a consequence a simple twist of one of the ends of the flux tube will not only give rise to an electric current inside the tube but also to a co-axial return current just outside the tube. Thus, there will be no magnetic energy outside the flux tube connected with the current system. Consequently, little or no magnetic field and energy can be extracted from this region which leads to a suppression 
of the K-S instability. In contrast, the Alfvén kink instability benefits only by the internal magnetic energy in the flux tube. We therefore expect this instability and its extensions to be relevant here.

The double helix clearly represents a vital ingredient in the trunk theory presented. It is therefore of some interest to consider the question of how durable this structure is. As shown by the experiments (Sect. 4) the double helix is mechanically stable. But how steady is it from a plasma physical point of view? We know that both parts of the flux tube in the double helix intertwine, at the same time as the axial magnetic fluxes in the two parts are oppositely directed. Might this situation be favourable for magnetic merging to occur? When considering this question we can benefit from a well-known model of solar flares developed by Gold \& Hoyle (1960).

In an effort to understand the mecanisms behind solar flares Gold and Hoyle studied an arrangement of twisted magnetic flux tubes which has some features in common with our double helix. In their model the energy of the flare is stored in two twisted magnetic flux tubes which form loops in the chromosphere of the Sun. The roots of the loops are situated in the photosphere where horizontal, rotatory motions give rise to their twist. If the loops happen to be brought side by side by more or less random photospheric motions, the magnetic energy connected with the axial flux may be released as a result of magnetic merging. For this to happen, two important conditions must be fulfilled. In the first place, the axial magnetic fields in the flux tubes must be opposed for an annihilation of these fields to be possible at all. Secondly, it is essential that the sense of twist of the flux tubes is opposite for the energy to be released. The axial electric currents are then parallel and attractive, and hence promote a merging of the two tubes.

We now apply Golds and Hoyles arguments to our double helix mechanism also involving two flux tubes in close contact. We find that the first condition is indeed fulfilled since the magnetic flux tube runs in both directions in the double helix. The second condition, however, can never be fulfilled in the double helix. The two parts of the flux tube in the double helix are always twisted in the same sense, since they form a coherent unit. This implies that the two parts must repel each other. As a result of this, the merging process will be very inefficient, and much slowed down.

An important question to consider is how the twisted filaments, or magnetic flux tubes, once were formed. One possibility is that they are condensed relics from the primeval molecular cloud. In this view, twisted flux tubes should constitute a natural ingredient of many molecular clouds. Another possibility is that rather loose flux tubes might be twisted predominantly after the central hot stars were born, and the H II region started to develop. Strong dynamic forces are then at work, mainly in the form of prominent winds, which may promote twisting. The process might bear some resemblance to the twisting of magnetic flux tubes beneath the photosphere of the Sun, discussed by Babcock (1961).

The double helix mechanism, proposed to be active in the twisted elephant trunks, is actually an entirely general mechanism which should be active also in other environments where the conditions are favourable. One such environment might be the shell of supernova remnants where magnetized filaments are known to be frequently present (e.g. Woltjer 1958b). Inside the remnants there is an enhanced pressure driving the shell outwards. Images of e.g. the Crab Nebula, obtained with the HST (Hester et al. 1996b), show that the shell of the nebula consists of a widespread system of thin, bright filaments with abrupt, extended outgrowths. The outgrowths mostly seem to point towards the central parts of the nebula. Many of the outgrowths are built up by two or more subfilaments, and some show clear signs to be twisted (e.g. regions F, G and H in Hester et al. 1996b). The structure of several of the filamentary outgrowths in the Crab Nebula thus exhibits apparent similarities to the structure of the twisted elephant trunks. It is an appealing thought that also the filaments in supernova remnants may be subject to the action of the double helix mechanism.

Another example of an environment where the double helix mechanism may be active is the expanding bubble outlined by the North Celestial Pool Loop (Gahm 2002). From the loop a high galactic latitude molecular cloud, composed of MBM 27-30 (Magnani et al. 1985) "hangs down" like a finger (Fig. 10) pointing towards the centre of the loop (Pound \& Goodman 1997). The cloud is strongly filamentary, and shows clear signs of being twisted. The similarity to several of the twisted elephant trunks is striking.

\section{Conclusions}

Elephant trunks are dark, elongated clouds which are seen silhouetted against bright $\mathrm{H}$ II regions excited by luminous young stars. The trunks are generally far from featureless but contain a wealth of structures in the form of filaments and knots. We have focused the attention to the fact that the structure of many elephant trunks seems to be twisted into a helical geometry.

1. From our series of CCD $\mathrm{H} \alpha$-images collected at the NOT we have selected a few examples of elephant trunks. Some of these trunks are large and well-developed like the Wrench Trunk and the Stag-Beetle Trunk (Figs. 1 and 3) with a lot of structure, while others are smaller and more simple (Fig. 4). In the well-developed trunks there are signs of twists. At their base these trunks are connected with two legs which run out from the trunk in the form of a V being directed away from the exciting hot stars. Often the legs consist of bundles of filaments. The smaller trunks are mostly made up of only a dark blob. But also these trunks are generally connected to two legs forming a V. In all trunks discussed in this paper the extinction is maximum in their head.

2. Our main goal has been to develop a new theory of the formation of such elephant trunks. Earlier studies have suggested that trunks may be formed from inhomogeneities in the primeval molecular cloud when influenced by the shell of an expanding H II region. Here, we have considered the likely situation that one or several filaments forming magnetic flux tubes in the molecular cloud are hit by the expanding shell. If there is a local mass condensation in the magnetic flux tube, and if further the magnetic field lines in the flux tube are not too twisted, the tube will take the shape of a V. Part of the less massive flanks of the tube will be captured by the shell while the mass condensation, due to its larger inertia, can not be accelerated so 


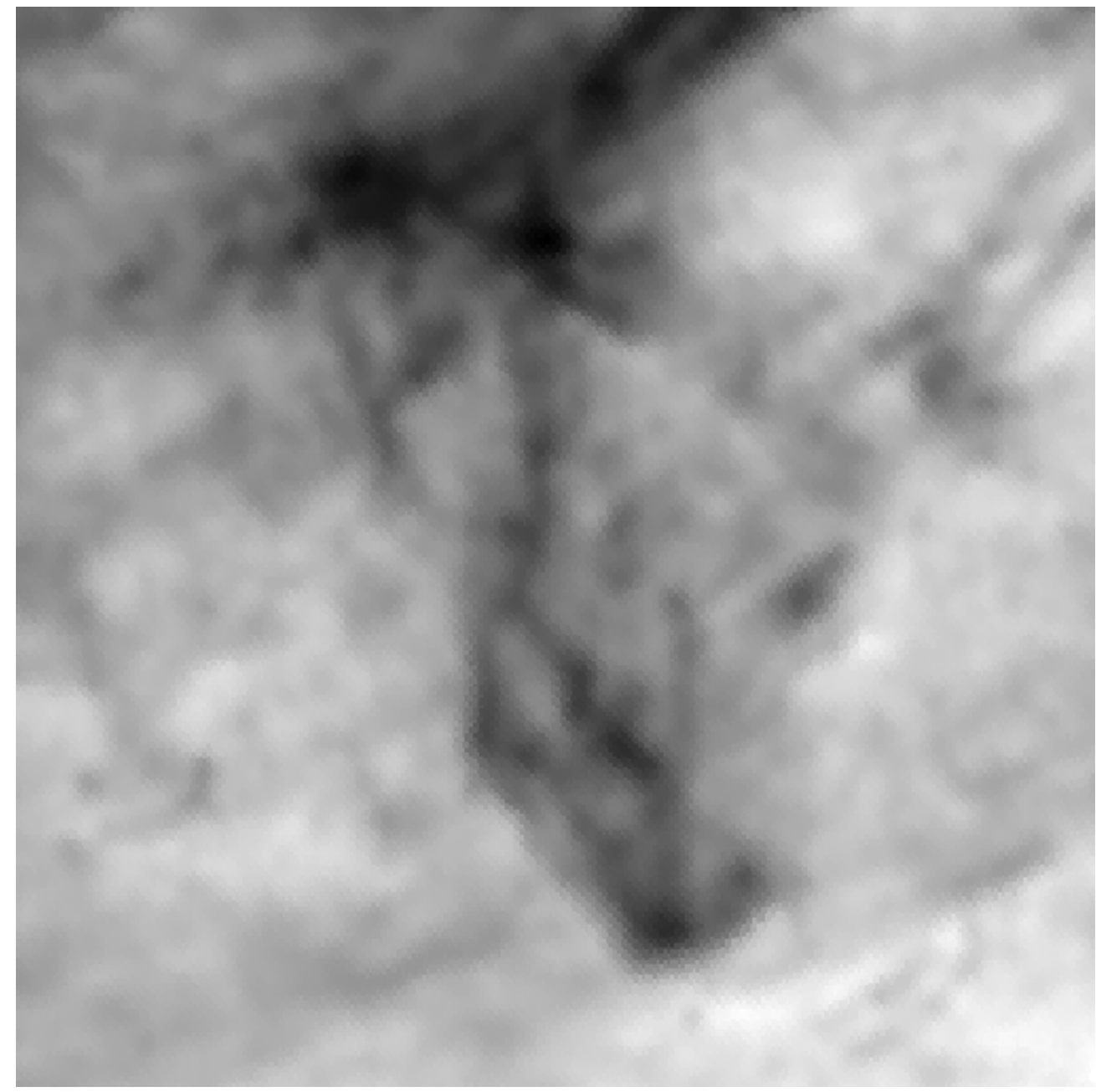

Fig. 10. Image of the high galactic latitude molecular cloud MBM 27-30 as captured by IRAS (SFD $100 \mu$ m). The cloud is strongly filamentary and appears to be twisted. In the upper parts of the frame the cloud connects to the filaments in the North Celestial Pole Loop. For the sake of clarity the image is shown as a negative. The image covers $6^{\circ} \times 6^{\circ}$ and is centered at $l=142^{\circ}, b=36^{\circ} .5$. Galactic north is up.

quickly, and hence will get behindhand. If the magnetic field lines in the flux tube are sufficiently twisted, those parts of the tube being most close to the apex of the $\mathrm{V}$ will be deformed into a double helix. The double helix is identified with the twisted elephant trunks like the Wrench Trunk or the Stag-Beetle Trunk (Figs. 1 and 3).

3. The physical processes involved in the theory of twisted trunks are fairly complex and intricate. In order to offer a more tangible description of the double helix mechanism we have developed a mechanical analogy model of the double helix mechanism. In this model the magnetic flux tube is replaced by a bundle of elastic strings while the inertia force of the mass condensation is replaced by the force of gravity acting on a weight attached to the middle of the bundle (Fig. 6). Experiments performed with the model show that if the bundle of strings is twisted by an angle that is smaller than a certain critical angle the bundle will take the shape of a $\mathrm{V}$ with the weight at the apex. If the bundle of strings is twisted beyond the critical angle the bundle will in addition form a double helix below the apex of the $\mathrm{V}$.

4. We have also developed a theoretical model of the double helix mechanism. The model comprises a twisted magnetic flux tube where a localized force is acting on the central part of the tube. Using the model we have performed a number of numerical calculations. The results of the calculations verify the existence of a critical value of the twist angle of the magnetic field lines. For twist angles larger than this value, part of the magnetic flux tube is deformed into a double helix. For twist angles smaller than the value the flux tube takes the shape of a $\mathrm{V}$ only. The numerical calculations also yield the geometrical conditions of the flux tube. Using a constant volume model we have been able to numerically simulate the analogy experiments fairly well.

5. By means of the theoretical model we have furthermore made numerical calculations applied to the Wrench Trunk. From the observed geometry of the trunk with its connecting legs we have found that the applied force (inertia force) should be of the order $1 \times 10^{21} \mathrm{~N}$ if the strength of the axial magnetic field component in an initially straight flux tube is $2 \times 10^{-9} \mathrm{~T}$. The flux tube considered has an estimated span of $\sim 8 \times 10^{16} \mathrm{~m}$, and is twisted some 17 to 18 turns. This twist together with the magnetic field corresponds to an electric current of $\sim 1 \times 10^{14} \mathrm{~A}$ in the tube. 
6. Both the Wrench Trunk and the Stag-Beetle Trunk have two jaws which protrude from their heads. Such jaws seem to be fairly common also in other trunks. We interpret the jaws to be the visible remains of the flux-tube loop which closes the double helix. Most of the gas and dust in the loop has been ionized by the intense radiation from the central hot stars, and is hence not visible any more, but the magnetic field must still close at the end of the double helix. At the tip of each of the jaws in the Wrench Trunk a small globule is about to break off due to the eroding action of the intense radiation. Also in the southern jaw of the Stag-Beetle Trunk there are a few outgrowths. We argue that these outgrowths are the remnants of especially dense filaments in the flux tube which have best withstanded the erosion caused by the radiation. We suggest that most of the EGGs observed in e.g. the Eagle Nebula are of the same nature.

7. The double helix mechanism is quite a general mechanism, and need not be active only in elephant trunks. Double helices should form also in other environments where the conditions are favourable. One such environment may be the supernova remnant. Another example is the expanding bubble outlined by the North Celestial Pole Loop (Fig. 10).

In the picture we have painted here of the formation of elephant trunks the magnetic field plays a decisive role in the shaping of the trunks. It is true that stellar winds from young, hot stars in the ambient $\mathrm{HII}$ region constitute the motive power. But it is the force of the twisted magnetic fields, assisted by radiation, that finally sculptures and chisels out the strange and beautiful shapes which are so characteristic of many elephant trunks.

Acknowledgements. The project was supported by the Swedish Natural Science Foundation and the Carl Trygger's Foundation.

\section{References}

Alfvén, H. 1950, Cosmical Electrodynamics (Oxford: Clarendon Press), pp. 82-84

Alfvén, H., \& Fälthammar, C.-G. 1963, Cosmical Electrodynamics (Oxford: Clarendon Press), edn. 2, pp. 117-119

Babcock, H. W. 1961, ApJ, 133, 572

Blitz, L., \& Thaddeus, P. 1980, ApJ, 241, 676

Carlqvist, P., Kristen, H., \& Gahm, G. F. 1998, A\&A, 332, L5

Carlqvist, P., Gahm, G. F., \& Kristen, H. 2002, Ap\&SS, 280, 405 (CGK02)

Di Bennedetto, G. P. 1997, ApJ, 486, 60
Faraday, M. 1839-1855, Experimental Researches in Electricity, 3266-3268

Frieman, E. A. 1954, ApJ, 120, 18

Gahm, G. F. 2003, in Recent Research Developments in Astrophysics, Research Signposts, in press

Garcia-Segura, G., \& Franco, J. 1996, ApJ, 469, 171

Guiliani, J. L. 1979, ApJ, 233, 280

Gold, T., \& Hoyle, F. 1960, MNRAS, 120, 89

Gyul'budagyan, A. L., \& Akopyan, V. A. 1990, Afz, 33, 395

Gyul'budagyan, A. L., Oganyan, G. B., \& Akhverdyan, L. G. 1994, Afz, 37, 577

Hensberge, H., Pavlovski, K., \& Vershueren, W. 2000, A\&A, 358, 553

Herbig, G. H. 1974, PASP, 86, 604

Hester, J. J., Scowen, P. A., Sankrit, R., et al. 1996a, AJ, 111, 2349

Hester, J. J., Stone, J. M., Scowen, P. A., et al. 1996b, ApJ, 456, 225

Heydari-Malayeri, M., Rosa, M. R., Zinnecker, H., et al. 1999, A\&A, 344,848

Israel, F. P., Johansson, L. E. B., Lequeux, J., et al. 1993, A\&A, 276, 25

Kadomtsev, B. B. 1966, Rev. Plasma Phys., 2, 153

Kadomtsev, B. B. 1992, Tokamak Plasma (London: IOP Publishing Ltd, Institute of Physics), pp. 34-35

Magnani, L., Blitz, L., \& Mundy, L. 1985, ApJ, 295, 402

Massey, P., Johnson, K. E., \& DeGiola-Eastwood, K. 1995, ApJ, 454, 151

McCaughrean, M. J. 1997, in Herbig-Haro Flows and the Birth of Low Mass Stars, ed. B. Reipurth, \& C. Bertout (Kluwer), Proc. IAU Symp., 182, 551

McCaughrean, M. J., \& Andersen, M. 2002, A\&A, 389, 513

Menon, T. K. 1962, ApJ, 135, 394

Minkowski, R. 1949, PASP, 61, 151

Moffatt, H. K. 1969, J. Fluid Mech., 35, 117

Moffatt, H. K., \& Proctor, M. R. E. 1985, J. Fluid Mech., 154, 493

Moffatt, H. K., \& Ricca, R. L. 1992, Proc. R. Soc. London A, 439, 411

Ogura, K., \& Sugitani, K. 1998, Pub. Astron. Soc. Australia, 15, 91

Osterbrock, D. E. 1957, ApJ, 125, 622

Pikel'ner, S. B. 1973, Comments on Astrophys., 5, 151

Pikel'ner, S. B., \& Sorochenko, R. L. 1974, SvA, 17, 443

Pilbratt, G. L., Altieri, B., Blommaert, J. A. D. L., et al. 1998, A\&A, 333, L9

Pottasch, S. 1956, Bull. Astron. Soc. Netherlands, 13, 77

Pound, M. W. 1998, ApJ, 493, L113

Pound, M. W., \& Goodman, A. A. 1997, ApJ, 482, 334

Schneps, M. H., Ho, P. T. P., \& Barrett, A. H. 1980, ApJ, 240, 84

Spitzer, L. Jr. 1954, ApJ, 120, 1

White, G. J., Nelson, R. P., Holland, W. S., et al. 1999, A\&A, 342, 233

Williams, J. P., Blitz, L., \& Stark, A. A. 1995, ApJ, 451, 252

Woltjer, L. 1958a, Proc. Natl. Acad. Sci. USA, 44, 489

Woltjer, L. 1958b, Bull. Astron. Soc. Netherlands, 14, 39 